\title{
La triade méthodologique: substance, énergie, information - comme fondement de la physiologie théorique
}

\author{
Victor Sahleanu \\ Université de Cluj et Institut d'Endocrinologie de Bucarest, Bucarest, Roumanie
}

\begin{abstract}
The methodological triad: substance, energy, information - a basis of a theoretical physiology. The organism as an open system and the organism as a system with an automatic adjustment, these are the two main contemporary schemes of theoretical physiology. However, these schemes, in spite of being synthetic in comparison to the models formerly proposed, are analytic according to the requirements of biological thinking. Some other schemes have to be integrated as strong heuristic means, for instance, the scheme of circuits generating oscillations of relaxation. The notions of "substance, energy, information" constitute a methodological triad which systematically can be used examining the great problems of life. "Energy" is a mediatory term which was to be recognized as possessing a working function and an informational one (as a signal). We propose to distinguish between structural and operational information on the one hand and between bounded and free (or brought up to date) information on the other hand. The living substance is "impregnated" with information, owing to a constant expense of energy. Adaptation, habituation, homeostasis, evolution - all these notions lend themselves to a more coherent analysis from the proposed point of view, as is demonstrated by examples.
\end{abstract}

\section{INTRODUCTION}

Si la biologie du XIXème siècle s'est développée autour des concepts de »cellule « et d'»évolution «, la biologie de notre temps a pris un nouvel essor grâce à l'introduction d'autres concepts fondamentaux et féconds. A notre avis, il faut souligner l'importance de trois notions: celle de système, celle de niveau d'organisation et celle d'information. Ce sont des notions plus abstraites que celles qui ont joué un grand rôle depuis le dernier siècle. Leur caractère plus abstrait reflète un trait caractéristique de la science de notre siècle, science qui est en grande partie théorique, quoique non purement spéculative. L'application conséquente de la pensée quantitative et de concepts mathématiques permettra d'établir des théories cohérentes de valeur heuristique. Chaque science théorique a besoin de concepts clairs et purs (on dirait pro analysi), de concepts pouvant faire l'objet d'un traitement mathématique ou impliquant une énumeration ou une mesure. C'est en ce sens que nous concevons une physiologie théorique comme étude systématique et exacte de l'organisation de la matière vivante au niveau de 
l'organisme dans le cadre important de la biologie théorique preconisée il y a 25 ans par L. v. BERTALANFFY (1942).

\section{SUBSTANCE, ENERGIE ET INFORMATION}

Le but de notre courte communication est d'attirer l'attention sur l'utilité, et d'envisager la nécessité de resystématiser - en vue d'une compréhension adéquate des faits fondée sur la logique intrinsèque des faits - les données et les problèmes de la physiologie, en tenant compte de la «triade»: substance, énergie, information. Ces trois concepts doivent acquérir, dans le domaine de la biologie, la fonction des «idées régulatrices et ordonnatrices» dans le sens kantien du mot.

Quoique l'appartenance exclusive d'une realité biologique au domaine d'une des parties de cette triade soit difficile à établir in concreto, il faut examiner tour-à-tour chaque fait sous les trois aspects distincts leur correspondant.

Le concept de «substance» ne pose aucun problème. Tout ce qui se rapporte au volume, à la masse, à la composition chimique, à la forme - dans ses aspects élémentaires -, à la croissance simple, est du ressort de ce domaine. C'est le domaine du métabolisme plastique par excellence ou celui des lésions anatomiques grossières qui entraînent la maladie. Le concept d' «énergie» est plus subtil. Il faut y tenir compte avant tout de la capacité d'effectuer un travail quelconque. L'énergétique d'une cellule ou d'un organe est étroitement liée à leur capacité à exécuter leur tâche specifique. Mais il faut aussi considérer des situations particulières, où une petite quantité d'énergie (ou de substance qui la contient) peut influencer de grandes mutations de substance ou d'énergie, grâce au dispositif d'amplification; leur signification est celle de signal ou de commande. C'est le rapport du type informationel, qui implique (1) la structuration de la substance des systèmes agissant les uns sur les autres (et aussi de l'énergie) et (2) le caractère adéquat de l'énergie mise en jeu, par rapport au système dynamique influencé. La lumière du soleil constitue «l'énergie - capacité de travail» pour les chloroplastes et «l'énergie information» pour la rétine. Le domaine informationnel est - par exemple - celui des régulations nerveuses ou hormonales.

La cybernétique est centrée autour du concept d'information et autour de celui de réglage. Il va de soi qu'il n'y a pas (ou presque pas) de rapports naturels du type informationnel en dehors de la vie. Mais cela ne revient pas à dire que toute la biologie doit être annexée au domaine de la cybernétique, pour la simple raison que la vie nous intéresse et importe aussi par ses côtés substantiels et énergétiques.

Prenons, comme premier exemple, le phénomène de l'accoutumance. On peut s'adapter à un toxique, à un rayonnement calorique excessif, à une source sonore monotone. Les mécanismes responsables de cette adaptation sont différents dans ces trois cas, mais les lois régissant ce phénomène, sont-elles les mêmes? C’est peu probable, mais il faut les étudier séparément. Prenons, comme un autre exemple, la notion même de substance constitutive de l'organisme. On doit distinguer les matières à rôle surtout plastique, les composés servant à produire de l'énergie, des substances messagères comme l'acéryle-choline ou les hormones. Le transfert qui s'opère dans les processus métaboliques peut être conçu d'un point de vue classique comme transfert de groupes 
ou de radicaux. La bioénergétique y ajoute la notion de transfert de charge (MULLIKEN) et la biologie moléculaire celle de transfert d'information s'opérant par exemple entre l'ADN et l'ARN, ou entre l'ARN et la protéine spécifique synthétisée. L'évolution de la vie sur la terre, elle-même, peut être conçue parallèlement comme évolution morphologique, comme évolution de l'énergogenèse et du rendement, comme évolution de l'intégration fonctionnelle et écologique. Une conclusion paraît surgir d'une telle analyse du processus de l'évolution: le perfectionnement informationnel prend le dessus. L'évolution da système nerveux devient de plus en plus essentielle; l'évolution morphologique du système nerveux se prolonge dans son évolution fonctionnelle, instinctuelle et psychique; les reflexes conditionnels et le psychisme s'acquittent mieux que les modifications morphologiques de la tâche de parfaire aux sollicitations du milieu. Il y a aussi, dans cette prééminence accordée à l'information, la capacité de faire face aux exigences d'un principe d'autonomie (l'homéostasie) ainsi que d'un principe d'économie. $\mathrm{La}$ «matrice» d'acide nucléique et les enzymes régissant la synthèse d'une protéine conservent de la substance (synthèse parfaitement orientée, du point de vue stéréochimique aussi, sans déchets), de l'énergie (augmentation du nombre des «chocs utiles» entre les molécules des réactants, réduction de l'énergie d'activation, etc.), du temps (la première molécule protéique "vivante» s'est produite il y a quelques milliards d'années, à la fin de nombreux tâtonnements pendant des ères géologiques). L'évolution humaine, essentiellement informationnelle, a changé, en $10^{6}$ ans, la face du monde, et la solution. «mentale» (ou sur modèles) d'un problème quelconque s'est substituée, dans la vie et dans l'activité de l'homme, à la solution vécue par l'expérience dans le monde extérieur à grands frais de matériel et d'énergie.

Aussi faut-il bien comprendre que le réglage n'est pas toujours un réglage informationnel. Du point de vue de la théorie des systèmes, aussi bien que du point de vue substantiel et thermodynamique, l'organisme ou la cellule sont des systèmes ouverts. Les transformations élémentaires de la matière obéissent à des principes de conservation de l'équilibre dynamique, ou du steady state (Fließgleichgerericht), qui peuvent se passer $\mathrm{du}$ concept d'information. La loi de l'action des masses, le principe de Le Chatelier, la notion d'équifinalité (v. BertalanfFy) en reflètent certains aspects. Ce sont aussi des systèmes énergétiques; les principes cités plus haut peuvent être déduits de certains principes thermodynamiques. Prenons, comme autre exemple, les systèmes disposant d'oscillations de relaxation (Kippschwingungen). Ici il y a une phase d'accumulation suivie d'une décharge brusque. Le système est dirigé par un réglage automatique - mais la notion d'information, néanmoins, n'est pas nécessaire pour en décrire le comportement.

Toutefois, il ne faut pas perdre de vue les relations de réciprocité entre les éléments de cette triade. On doit reconnaître premièrement, la substratation métabolique de chaque acte vital plastique, énergétique ou informationnel. On doit tenir compte que la structure spatiale (Gefüge ou Gestalt) d'un système ouvert à compartimentation interne, offre de ce chef des «zones stratégiques» (par exemple des membranes) où peuvent s'exercer les actions du type informationnel. On doit se convaincre que chaque structure vitale complexe se forme et se maintient grâce à un réglage de type cybernétique et qu'elle consomme de l'énergie. Il faut étudier aussi le reflet substantiel et énergétique d'une action surtout informationnelle (il semble, par exemple, que l'apprentissage ne se déroule pas sans modifications biochimiques du sang et du cerveau, etc.). 
Ce sont, certainement, des problèmes où l'explication tient du domaine de la biologie moléculaire, parce que ce sont sans aucun doute les macromolécules biologiques qui jouent un rôle tant dans la plastique que dans l'énergétique ou la cybernétique biologiques. Efficacité et specificité enzymatiques, libération, transfert et transformation des énergies contenues dans l'espace qui leur est voisin, sensibilité très sélective, amplification des effets subis - voilà des traits qu'on pourrait encore approfondir. L'inhibition de l'activité enzymatique par le produit final de la réaction, est-elle peut-être aussi le schéma le plus simple d'un feed-back informationnel?

Dans l'histoire de la pensée scientifique, l'énergétique s'est développée après l'étude de la «substance», la cybernétique après l'étude de l'énergie. Le fait d'être conscient de la nécessité d'aborder les trois aspects de chaque nouveau problème biologique, «raccourcira» de beaucoup la voie vers la solution. C’est pour cela que nous avons souligné l'importance de la triade et que nous l'avons conçue comme appartenant à la méthodologie, c'est-à-dire à la manière de penser scientifiquement.

\section{RESUME}

1. La biologie actuelle tend à se resystématiser autour de trois notions modernes: celle de système, celle de niveau d'organisation et celle d'information.

2. Pour concevoir les faits de façon adéquate, il est indispensable de resystématiser les données et les problèmes de la physiologie en tenant compte de la triade: substance, énergie, information.

3. Les rapports du type informationnel impliquent: la structuration de la substance des systèmes agissant les uns sur les autres aussi que de l'énergie; le caractère adéquat de l'énergie mise en jeu par rapport au système dynamique influencé; l'amplification.

4. En même temps l'organisme doit être conçu comme système ouvert, comme oscillateur à oscillations de relaxation et comme système à réglage automatique. Le réglage n'est pas toujours un réglage informationnel.

\section{LITTÉRATURE CITEEE}

BertalanfFy, L. v., 1942. Theoretische Biologie. Bd 2. Stoffwechsel, Wachstum. Borntraeger, Berlin, 362 pp.

Mulliken, R. S. \& Rieke, C. A., 1941. Molecular electronic spectra, dispersion and polarization, Rep. Prog. Phys, 8, 231. 